\title{
Current Status of Ornithofauna of Ambedkar Nagar, Uttar Pradesh, India
}

\author{
Akhilesh Kumar ${ }^{1}$, Sonika Kushwaha ${ }^{1}$ * \\ ${ }^{1}$ Indian Biodiversity Conservation Society, Khailar, BHEL, Jhansi, U.P., India
}

*Address for Correspondence: Indian Biodiversity Conservation Society, 1474, Near- Sarvodaya Petrol Pump, Indira Nagar, Khailar BHEL, Jhansi-284120, Uttar Pradesh, India

E-mail: ibcsforall@gmail.com, sonika2107@gmail.com

Received: 11 Oct 2018/ Revised: 17 Jan 2019/ Accepted: 15 Apr 2019

\begin{abstract}
Background: The bird diversity in many districts of Uttar Pradesh is still unexplored. Information on baseline data of species can be used to set priorities, allowing conservation effort to be focused on those species that need the most attention. This study was therefore undertaken to investigate the Ornithfauna of Ambedkar Nagar.

Methods: Extensive field surveys were undertaken in all the three season i.e. rainy, summer and winter. Line transects and point count methods were used for the bird counting.

Results: During the three years of study (November 2015-December 2018), a diverse variety of 170 bird species was discovered. The highest bird species belonged to family Passeridae (13) followed by Anatidae (12), Corvidae (12) and Muscicapidae (11). However, no particular site was discovered that could be identified as a birding spot in the district. The lakes such as Darvan, Hanswar, Devhat have the potential to support rich avifaunal diversity however they were under serious threats due to various anthropogenic activities.

Conclusion: The study reflects the potential of Ambedkar Nagar to support a rich diversity of ornithofauna. There was a need to reduce anthropogenic mortality of birds or to educate the public to support for and to implement remedial measures. The organization of bird watching events on various occasions such as World Wetlands Day, International Day for Biological Diversity and World Wildlife Week will draw the intention and interest of local people and youth. Further studies based on the ecology of threatened and endangered birds are needed.
\end{abstract}

Key-words: Anthropogenic, Diversity, Threats, Mortality, Ornithofauna

\section{INTRODUCTION}

As far as bird diversity is concerned, India is a blessed country. It has more than 1300 bird species which is over $13 \%$ of the world's bird species ${ }^{[1]}$. Uttar Pradesh has a rich and varied Ornithofauna of over 550 species ${ }^{[2]}$. Still, the bird diversity in many districts of Uttar Pradesh is still unexplored. This includes Ambedkar Nagar, a district in the Ayodhya division. Ambedkar Nagar was created on September 29, 1995, and was named in the memory of Dr. Bhim Rao Ambedkar.

\section{How to cite this article}

Kumar A, Kushwaha S. Current Status of Ornithofauna of Ambedkar Nagar, Uttar Pradesh, India. SSR Inst. Int. J. Life Sci., 2019; 5(3): 2302-2316.
Ideally, the forest must be at least $33 \%$ of the total geographical area, but as far as the study area is concerned the forest cover condition is in the distressing state. In 1995 there was $0.24 \%$ forest cover, which got reduced to $0.14 \%$ of the total geographical area in 2012 . The situation was even worst in 2005 when the forest area in the district was just $0.11 \%{ }^{[3]}$. The wild animals found in the district are not remarkable for either their number or variety. The ordinary species which occur to the south of the river Ghaghra includes jackals, foxes, wild boar. Ambedkar Nagar has been traditionally an agriculturally dominated area. Being an agrarian economy, domestic animals hold an important place in society. The natural vegetation is replaced by mixed vegetation that includes wild varieties with groove plantations. Due to fast habitat destruction and fragmentation, urbanisation, loss of forest and another natural system, mining, drainage of swamps, and other 
Wet lands are reducing the potential habitat of many birds. Information on baseline data of species can be used to set priorities, allowing conservation effort to be focused on those species that need the most attention. This study was therefore undertaken to investigate the Ornithfauna of Ambedkar Nagar.

\section{MATERIALS AND METHODS}

Study Area- The district carved out from Faizabad is situated in the eastern part of the state of Uttar Pradesh. The River Tamasa (Tons) divides the city of Ambedkarnagar into the two parts Akbarpur and
Shahzadpur. Akbarpur is a city and a municipal board. The total area of the district is $2350.0 \mathrm{Sq}$. $\mathrm{Km}$. The rural area covers $2255.1 \mathrm{Sq}$. $\mathrm{Km}$. and urban recorded $94.9 \mathrm{Sq}$. $\mathrm{Km}$. It lies between $26^{\circ} 09^{\prime \prime} \mathrm{N}$ to $26^{\circ} 40^{\prime \prime} \mathrm{N}$ latitudes and $82^{\circ} 12^{\prime \prime} \mathrm{E}$ to $83^{\circ} 05^{\prime \prime} \mathrm{E}$ longitudes and bounded in the north by district Basti and Sant Kabir Nagar, in north-east by Gorakhpur, in south by Sultanpur, in the west by Faizabad (Ayodhya) and in the east by district Azamgarh (Fig. 1). The district is divided into nine development blocks namely Akbarpur, Katehri, Bhiti, Tanda, Baskhari, Ramnagar, Jalalpur, Jahangirganj and Bhiyaon ${ }^{[4]}$.

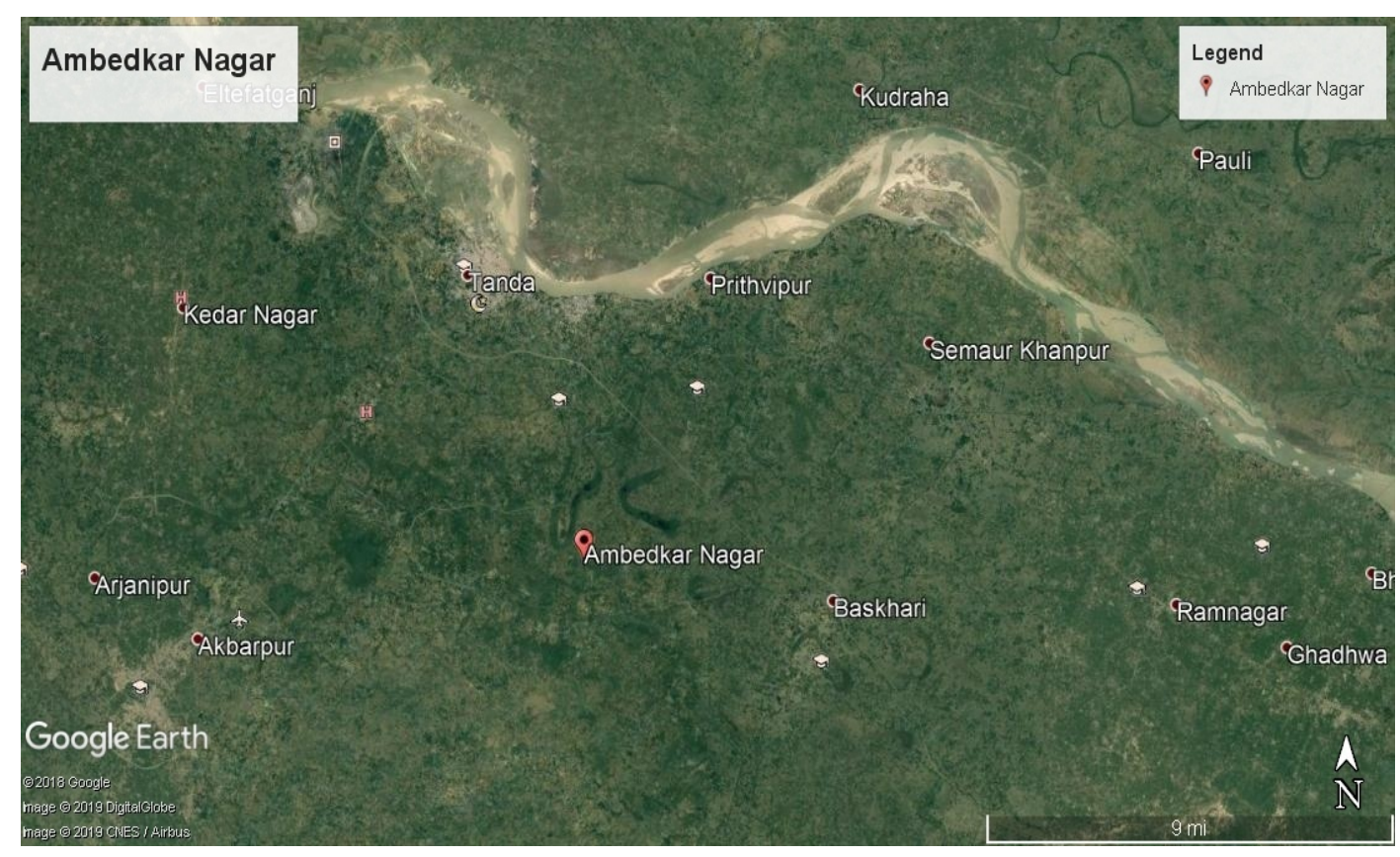

Fig. 1: Study Area (Source: GoogleEarth)

The study area has a number of rivers and streams. The principal rivers are the Ghagra, Tons and Majohi. There are numerous large and small lakes spread over the district like Devhat, Hanswar, and Darvan. The climatic condition of the district resembles that of eastern Uttar Pradesh that is characterized by a rhythm of seasons classified into winter Season (November to February), summer season (March to Mid June) and the rainy season (Mid June to October). The temperature in the summer season up to $45^{\circ} \mathrm{C}$, while in winter temperature drops down up to $4^{\circ} \mathrm{C}$. The average annual rainfall of the district was $1135.5 \mathrm{~mm}{ }^{[5]}$.
To study the avifauna of Ambedkar Nagar an extensive survey of all the nine blocks of the district was done in all the three seasons i.e. rainy, summer and winter from November 2015 to December 2018. The birds were observed during the most active and specific time period of the day, i.e., early morning from 06:00 to 09:00 hours and in the evening from 16:00 to $18: 00$ hours in the summer while 07:00 to 10:00 hrs in the morning and 15:00 to $17: 00 \mathrm{hrs}$ in the winter. Line transects and points count methods were described by Verner ${ }^{[6]}$ and Bibby et al. ${ }^{[7]}$ were used for the bird counting. A transect of 50 metre length was selected and a 50-metre wide strip on each side of the transect was selected for bird counting. At each transect, birds were counted using a $10 \times 50 \mathrm{~mm}$ binocular, based on their morphological 
characteristics such as beak shape and colour, type of foot, the colour of shank, feathers, foot, and size of birds. Identification was carried out using standard literature ${ }^{[1,8,9]}$. For more authentications of species, photographs of birds were taken using 70 DSLR Camera. Furthermore, recorded birds were categorised according to their IUCN status such NA-Not Assessed, LC-Least Concern, NT-Near Threatened, V-Vulnerable, EEndangered, CE-Critically Endangered. Based on the frequency of field observation, the abundance of birds was categorized as Common, Fairly common, Uncommon and Rare. Questionnaire surveys were also made in the nearby villages and surrounding of the selected points.

\section{RESULTS}

On compiling the data it was found that the district has 170 bird species belonging to 48 families (Table 1). The highest bird species belonged to family Passeridae (13) followed by Anatidae (12), Corvidae (12) and Muscicapidae (11). Only 1 or 2 species were recorded in 26 families (Fig. 2).

Table 1: List of bird species in Ambedkar Nagar

\begin{tabular}{|c|c|c|c|c|c|}
\hline S. No. & Common name & Scientific name & $\begin{array}{l}\text { Abundance } \\
\text { Code }\end{array}$ & Family & $\begin{array}{l}\text { IUCN } \\
\text { Status }\end{array}$ \\
\hline 1. & Jungle bush quail & Perdicula asiatica & UC & \multirow{3}{*}{ Phasianidae } & LC \\
\hline 2. & Grey Francolin & Francolinus pondicerianus & C & & LC \\
\hline 3. & Indian Peafowl & Pavo cristatus & C & & LC \\
\hline 4. & $\begin{array}{l}\text { Lesser Whistling } \\
\text { Duck }\end{array}$ & Dendrocygna javanica & C & Dendrocygnidae & LC \\
\hline 5. & Grey Lag Goose & Anser anser & C & \multirow{12}{*}{ Anatidae } & LC \\
\hline 6. & $\begin{array}{l}\text { Bar Headed } \\
\text { Goose }\end{array}$ & Anser indicus & C & & LC \\
\hline 7. & Ruddy Shelduck & Tadorna ferruginea & C & & LC \\
\hline 8. & Comb Duck & Sarkidiornis melanotos & FC & & LC \\
\hline 9. & Gadwal & Anas strepera & C & & LC \\
\hline 10. & Eurasian Wigeon & Anas penelope & C & & LC \\
\hline 11. & Spot Billed Duck & Anas poecilorhyncha & C & & LC \\
\hline 12. & $\begin{array}{l}\text { Northern } \\
\text { Shoveler }\end{array}$ & Anas clypeata & C & & LC \\
\hline 13. & Northern Pintail & Anas acuta & C & & LC \\
\hline 14. & Common Teal & Anas crecca & C & & LC \\
\hline 15. & $\begin{array}{l}\text { Red Crested } \\
\text { Pochard }\end{array}$ & Rhodonessa rufina & FC & & LC \\
\hline 16. & $\begin{array}{l}\text { Common } \\
\text { Pochard }\end{array}$ & Aythya ferina & C & & LC \\
\hline 17. & $\begin{array}{c}\text { Small } \\
\text { Buttonquail }\end{array}$ & Turnix sylvatica & UC & \multirow{2}{*}{ Turnicidae } & LC \\
\hline 18. & $\begin{array}{c}\text { Barred } \\
\text { Buttonquail }\end{array}$ & Turnix suscitator & C & & LC \\
\hline 19. & $\begin{array}{l}\text { Eurasian } \\
\text { Wryneck }\end{array}$ & Jynx torquilla & FC & Picidae & LC \\
\hline
\end{tabular}




\begin{tabular}{|c|c|c|c|c|c|}
\hline S. No. & Common name & Scientific name & $\begin{array}{l}\text { Abundance } \\
\text { Code }\end{array}$ & Family & $\begin{array}{r}\text { IUCN } \\
\text { Status }\end{array}$ \\
\hline & Brown Capped & & & & \\
\hline 20. & Pygmy & Dendrocopos nanus & FC & & LC \\
\hline & Woodpecker & & & & \\
\hline 21. & $\begin{array}{l}\text { Black Rumped } \\
\text { Flameback }\end{array}$ & Dinopium benghalense & C & & LC \\
\hline 22. & $\begin{array}{l}\text { Brown Headed } \\
\text { Barbet }\end{array}$ & Megalaima zeylanica & FC & & NA \\
\hline 23. & $\begin{array}{l}\text { Coppersmith } \\
\text { Barbet }\end{array}$ & Megalaima haemacephala & C & Megalaımıdae & LC \\
\hline 24. & $\begin{array}{l}\text { Indian Grey } \\
\text { Hornbill }\end{array}$ & Ocyceros birostris & FC & Bucerotidae & LC \\
\hline 25. & $\begin{array}{l}\text { Common } \\
\text { Hoopoe }\end{array}$ & Upupa epops & C & Upupidae & LC \\
\hline 26. & Indian Roller & Coracias benghalensis & C & Coraciidae & LC \\
\hline 27. & $\begin{array}{l}\text { Common } \\
\text { Kingfisher }\end{array}$ & Alcedo atthis & FC & Alcedinidae & LC \\
\hline 28. & $\begin{array}{l}\text { White Throated } \\
\text { Kingfisher }\end{array}$ & Halcyon smyrnensis & C & & LC \\
\hline 29. & $\begin{array}{l}\text { Stork-billed } \\
\text { Kingfisher }\end{array}$ & Halcyon capensis & C & Halcyonidae & LC \\
\hline 30. & Pied Kingfisher & Ceryle rudis & C & Cerylidae & LC \\
\hline 31. & Green Bee Eater & Merops orientalis & C & Meropidae & LC \\
\hline 32. & Pied Cuckoo & Clamator jacobinus & FC & & LC \\
\hline 33. & $\begin{array}{l}\text { Common Hawk } \\
\text { Cuckoo }\end{array}$ & Hierococcyx varius & FC & Cuculidae & LC \\
\hline 34. & Asian Koel & Eudynamys scolopacea & C & & LC \\
\hline 35. & Sirkeer Malkoha & $\begin{array}{l}\text { Phaenicophaeus } \\
\text { leschenaultii }\end{array}$ & UC & & LC \\
\hline 36. & Greater Coucal & Centropus sinensis & C & Centropodidae & LC \\
\hline 37. & $\begin{array}{l}\text { Alexandrine } \\
\text { Parakeet }\end{array}$ & Psittacula eupatria & FC & & LC \\
\hline 38. & $\begin{array}{l}\text { Rose Ringed } \\
\text { Parakeet }\end{array}$ & Psittacula krameri & C & Psittacidae & LC \\
\hline 39. & $\begin{array}{l}\text { Plum Headed } \\
\text { Parakeet }\end{array}$ & Psittacula cyanocephala & FC & & LC \\
\hline 40. & House Swift & Apus affinis & C & Apodidae & LC \\
\hline 41. & $\begin{array}{l}\text { Collared Scops } \\
\qquad \text { Owl }\end{array}$ & Otus bakkamoena & FC & & LC \\
\hline 42. & Brown Fish Owl & Ketupa zeylonsis & UC & Strigidae & LC \\
\hline 43. & Barn owl & Tyto alba & UC & & LC \\
\hline
\end{tabular}




\begin{tabular}{|c|c|c|c|c|c|}
\hline S. No. & Common name & Scientific name & $\begin{array}{l}\text { Abundance } \\
\text { Code }\end{array}$ & Family & $\begin{array}{l}\text { IUCN } \\
\text { Status }\end{array}$ \\
\hline 44. & $\begin{array}{l}\text { Mottled wood } \\
\text { owl }\end{array}$ & Strix ocellata & UC & & LC \\
\hline 45. & Jungle Owlet & Glaucidium radiatum & FC & & LC \\
\hline 46. & Spotted Owlet & Athene brama & FC & & LC \\
\hline 47. & Rock Pigeon & Columba livia & C & \multirow{6}{*}{ Columbidae } & LC \\
\hline 48. & Laughing Dove & Streptopelia senegalensis & C & & LC \\
\hline 49. & Spotted Dove & Streptopelia chinensis & C & & LC \\
\hline 50. & $\begin{array}{l}\text { Eurasian Collard } \\
\text { Dove }\end{array}$ & Streptopelia decaocto & C & & LC \\
\hline 51. & $\begin{array}{l}\text { Red Collared } \\
\text { Dove }\end{array}$ & Streptopelia tranquebarica & FC & & LC \\
\hline 52. & $\begin{array}{l}\text { Yellow Footed } \\
\text { Green Pigeon }\end{array}$ & Treron phoenicoptera & C & & LC \\
\hline 53. & Sarus Crane & Grus antigon & UC & Gruidae & NT \\
\hline 54. & $\begin{array}{l}\text { White Breasted } \\
\text { Waterhen }\end{array}$ & Amaurornis phoenicurus & C & \multirow{4}{*}{ Rallidae } & LC \\
\hline 55. & $\begin{array}{c}\text { Purple } \\
\text { Swamphen }\end{array}$ & Porphyrio porphyrio & C & & LC \\
\hline 56. & $\begin{array}{l}\text { Common } \\
\text { Moorhen }\end{array}$ & Gallinula chloropus & C & & LC \\
\hline 57. & Common Coot & Fulica atra & C & & LC \\
\hline 58. & Common snipe & Gallinago gallinago & FC & \multirow{6}{*}{ Scolopacidae } & LC \\
\hline 59. & $\begin{array}{l}\text { Common } \\
\text { Redshank }\end{array}$ & Tringa totanus & C & & LC \\
\hline 60. & $\begin{array}{c}\text { Common } \\
\text { GreenShank }\end{array}$ & Tringa nebularia & C & & LC \\
\hline 61. & Green Sandpiper & Tringa ochropus & FC & & LC \\
\hline 62. & Wood Sandpiper & Tringa glareola & C & & LC \\
\hline 63. & $\begin{array}{l}\text { Common } \\
\text { Sandpiper }\end{array}$ & Actitis hypoleucos & C & & LC \\
\hline 64. & $\begin{array}{l}\text { Pheasant tailed } \\
\text { jacana }\end{array}$ & Hydrophasianus chirurgus & C & \multirow{2}{*}{ Jacanidae } & LC \\
\hline 65. & $\begin{array}{l}\text { Bronze Winged } \\
\text { Jacana }\end{array}$ & Metopidius indicus & C & & LC \\
\hline 66. & $\begin{array}{l}\text { Eurasian Thick } \\
\text { Knee }\end{array}$ & Burhinus oedicnemus & C & Burhinidae & LC \\
\hline 67. & $\begin{array}{c}\text { Black Winged } \\
\text { Stilt }\end{array}$ & Himantopus himantopus & C & \multirow{2}{*}{ Charadriidae } & LC \\
\hline 68. & $\begin{array}{l}\text { Little Ringed } \\
\text { Plover }\end{array}$ & Charadrius dubius & C & & LC \\
\hline
\end{tabular}




\begin{tabular}{|c|c|c|c|c|c|}
\hline S. No. & Common name & Scientific name & $\begin{array}{l}\text { Abundance } \\
\text { Code }\end{array}$ & Family & $\begin{array}{l}\text { IUCN } \\
\text { Status }\end{array}$ \\
\hline 69. & $\begin{array}{l}\text { Yellow Wattled } \\
\text { Lapwing }\end{array}$ & Vanellus malarbaricus & UC & & NA \\
\hline 70. & River Lapwing & Vanellus duvaucelii & FC & & NT \\
\hline 71. & $\begin{array}{l}\text { Red Wattled } \\
\text { Lapwing }\end{array}$ & Vanellus indicus & C & & LC \\
\hline 72. & Small Pratincole & Glareola lactea & FC & Glareolidae & LC \\
\hline 73. & River tern & Sterna aurantia & FC & Laridae & NT \\
\hline 74. & $\begin{array}{l}\text { Oriental Honey } \\
\text { Buzzard }\end{array}$ & Pernis ptilorhyncus & FC & & LC \\
\hline 75. & Osprey & Pandion haliaetus & FC & & LC \\
\hline 76. & $\begin{array}{l}\text { Black Shouldered } \\
\text { Kite }\end{array}$ & Elanus caeruleus & FC & & LC \\
\hline 77. & Black Kite & Milvus migrans & C & Accinitridae & LC \\
\hline 78. & Egyptian Vulture & Neophron perconpterus & FC & & $\mathrm{E}$ \\
\hline 79. & Shikra & Accipiter badius & C & & LC \\
\hline 80. & $\begin{array}{l}\text { White Eyed } \\
\text { Buzzard }\end{array}$ & Butastur teesa & FC & & LC \\
\hline 81. & $\begin{array}{l}\text { Eurasian Marsh } \\
\text { harrier }\end{array}$ & Circus aeruginosus & C & & LC \\
\hline 82. & Little Grebe & Tachybaptus ruficollis & C & Podicipedidae & LC \\
\hline 83. & Darter & Anhinga melanogaster & FC & Anhingidae & NT \\
\hline 84. & Little Cormorant & Phalacrocorax niger & C & Phalacrocoracidae & LC \\
\hline 85. & $\begin{array}{c}\text { Indian } \\
\text { Cormorant }\end{array}$ & Phalacrocorax fuscicollis & C & & LC \\
\hline 86. & Great Cormorant & Phalacrocorax carbo & FC & & LC \\
\hline 87. & Purple Heron & Ardea purpurea & C & & LC \\
\hline 88. & Grey Heron & Ardea cinerea & C & & LC \\
\hline 89. & Little Egret & Egretta gargetta & C & & LC \\
\hline 90. & Great Egret & Casmerodius albus & C & & LC \\
\hline 91. & $\begin{array}{l}\text { Intermediate } \\
\text { Egret }\end{array}$ & Mesophoyx intermedia & C & Ardeidae & LC \\
\hline 92. & Cattle Egret & Bubulcus ibis & C & & LC \\
\hline 93. & $\begin{array}{l}\text { Indian Pond } \\
\text { Heron }\end{array}$ & Ardeola grayii & C & & LC \\
\hline 94. & Little Heron & Butorides straitus & UC & & LC \\
\hline 95. & $\begin{array}{l}\text { Black Crowned } \\
\text { Night Heron }\end{array}$ & Nycticorax nycticorax & FC & & LC \\
\hline
\end{tabular}




\begin{tabular}{|c|c|c|c|c|c|}
\hline S. No. & Common name & Scientific name & $\begin{array}{l}\text { Abundance } \\
\text { Code }\end{array}$ & Family & $\begin{array}{l}\text { IUCN } \\
\text { Status }\end{array}$ \\
\hline 96. & Black Ibis & Pseudibis papillosa & FC & \multirow{3}{*}{ Threskiornithidae } & LC \\
\hline 97. & Glossy ibis & Plegadis falcinellus & FC & & LC \\
\hline 98. & Black-headed ibis & $\begin{array}{c}\text { Threskiornis } \\
\text { melanocephalus }\end{array}$ & FC & & NT \\
\hline 99. & Painted Stork & Mycteria leucocephala & C & \multirow{5}{*}{ Ciconiidae } & NT \\
\hline 100. & Asian Openbill & Anastomus oscitans & FC & & LC \\
\hline 101. & $\begin{array}{l}\text { Woolly Necked } \\
\text { Sork }\end{array}$ & Ciconia episcopus & FC & & V \\
\hline 102. & $\begin{array}{l}\text { Black Necked } \\
\text { Stork }\end{array}$ & Ephippiorhynchus asiaticus & C & & NT \\
\hline 103. & Lesser Adjutant & Leptoptilos javanicus & UC & & NT \\
\hline 104. & $\begin{array}{l}\text { Long Tailed } \\
\text { Shrike }\end{array}$ & Lanius schach & C & \multirow{2}{*}{ Laniidae } & LC \\
\hline 105. & $\begin{array}{l}\text { Bay-backed } \\
\text { Shrike }\end{array}$ & Lanius vittatus & FC & & LC \\
\hline 106. & Rufous Treepie & Dendrocitta vagabunda & C & \multirow{12}{*}{ Corvidae } & LC \\
\hline 107. & House Crow & Corvus splendens & C & & LC \\
\hline 108. & Large Billed Crow & Corvus macrorhynchos & c & & LC \\
\hline 109. & $\begin{array}{l}\text { Eurasian Golden } \\
\text { Oriole }\end{array}$ & Oriolus oriolus & C & & LC \\
\hline 110. & $\begin{array}{l}\text { Black Hooded } \\
\text { Oriole }\end{array}$ & Oriolus xanthornus & FC & & LC \\
\hline 111. & $\begin{array}{l}\text { Large Cuckoo } \\
\text { shrike }\end{array}$ & Coracina macei & UC & & LC \\
\hline 112. & Small Minivet & Pericrocotus cinnamomeus & FC & & LC \\
\hline 113. & $\begin{array}{l}\text { White-browed } \\
\text { fantail }\end{array}$ & Rhipidura aureola & FC & & LC \\
\hline 114. & Black Drongo & Dicrurus macrocercus & C & & LC \\
\hline 115. & Common Iora & Aegithina tiphia & FC & & $\mathrm{LC}$ \\
\hline 116. & $\begin{array}{c}\text { Common } \\
\text { Woodshrike }\end{array}$ & $\begin{array}{l}\text { Tephrodornis } \\
\text { pondicerianus }\end{array}$ & FC & & LC \\
\hline 117. & $\begin{array}{l}\text { Asian Paradise- } \\
\text { flycatcher }\end{array}$ & Terpsiphone paradisi & FC & & LC \\
\hline 118. & $\begin{array}{l}\text { Oriental Magpie } \\
\text { Robin }\end{array}$ & Copsychus saularis & C & \multirow{5}{*}{ Muscicapidae } & LC \\
\hline 119. & Indian Robin & Saxicoloides fulicata & C & & LC \\
\hline 120. & Black Redstart & Phoenicurus ochruros & FC & & LC \\
\hline 121. & Pied Bushchat & Saxicola caprata & C & & $\mathrm{LC}$ \\
\hline 122. & Brown Rock Chat & Cercomela fusca & FC & & LC \\
\hline
\end{tabular}




\begin{tabular}{|c|c|c|c|c|c|}
\hline S. No. & Common name & Scientific name & $\begin{array}{l}\text { Abundance } \\
\text { Code }\end{array}$ & Family & $\begin{array}{l}\text { IUCN } \\
\text { Status }\end{array}$ \\
\hline 123. & $\begin{array}{l}\text { Common } \\
\text { Stonechat }\end{array}$ & Saxicola torquata & C & & LC \\
\hline 124. & $\begin{array}{l}\text { Red-throated } \\
\text { flycatcher }\end{array}$ & Ficedula parva & FC & & LC \\
\hline 125. & $\begin{array}{l}\text { Verditer } \\
\text { flycatcher }\end{array}$ & Eumyias thalassina & FC & & LC \\
\hline 126. & $\begin{array}{l}\text { Tickell's Blue } \\
\text { flycatcher }\end{array}$ & Cyornis tickelliae & FC & & LC \\
\hline 127. & $\begin{array}{c}\text { Grey-headed } \\
\text { canary flycatcher }\end{array}$ & Culicicapa ceylonensis & FC & & LC \\
\hline 128. & Bluethroat & Luscinia svecica & FC & & LC \\
\hline 129. & $\begin{array}{l}\text { Brahminy } \\
\text { Starling }\end{array}$ & Sturnus pagodarum & C & & LC \\
\hline 130. & $\begin{array}{l}\text { Chestnut-tailed } \\
\text { Starling }\end{array}$ & Sturnus malabaricus & FC & & LC \\
\hline 131. & Jungle mynah & Acridotheres fuscus & FC & Sturnidae & LC \\
\hline 132. & $\begin{array}{l}\text { Asian Pied } \\
\text { Starling }\end{array}$ & Sturnus contra & C & & LC \\
\hline 133. & Common Mynah & Acridotheres ginginianus & C & & LC \\
\hline 134. & Bank Mynah & Acridotheres ginginianus & C & & LC \\
\hline 135. & Great Tit & Parus major & FC & Paridae & LC \\
\hline 136. & Plain Martin & Riparia rupestris & FC & & LC \\
\hline 137. & Barn Swallow & Hirundo rustica & C & & LC \\
\hline 138. & $\begin{array}{l}\text { Wire Tailed } \\
\text { Swallow }\end{array}$ & Hirundo smithii & FC & Hirundinidae & LC \\
\hline 139. & $\begin{array}{l}\text { Streak-throated } \\
\text { swallow }\end{array}$ & Hirundo fluvicola & FC & & LC \\
\hline 140. & $\begin{array}{l}\text { Red Whiskered } \\
\text { Bulbul }\end{array}$ & Pycnonotus jocosus & C & & LC \\
\hline 141. & $\begin{array}{l}\text { Red Vented } \\
\text { Bulbul }\end{array}$ & Pycnonotus cafer & C & Pycnonotidae & LC \\
\hline 142. & Zitting Cisticola & Cisticola juncidis & FC & & LC \\
\hline 143. & $\begin{array}{l}\text { Grey Breasted } \\
\text { Prinia }\end{array}$ & Prinia hodgsonii & FC & Cisticolidae & LC \\
\hline 144. & Ashy Prinia & Prinia socialis & C & & LC \\
\hline 145. & Plain Prinia & Prinia inornata & C & & LC \\
\hline 146. & $\begin{array}{l}\text { Oriental White } \\
\text { Eye }\end{array}$ & Zosterops palpebrosus & C & Zosteropidae & LC \\
\hline 147. & $\begin{array}{l}\text { Common } \\
\text { Tailorbird }\end{array}$ & Orthotomus sutorius & C & Sylviidae & LC \\
\hline
\end{tabular}




\begin{tabular}{|c|c|c|c|c|c|}
\hline S. No. & Common name & Scientific name & $\begin{array}{l}\text { Abundance } \\
\text { Code }\end{array}$ & Family & $\begin{array}{l}\text { IUCN } \\
\text { Status }\end{array}$ \\
\hline 148. & $\begin{array}{l}\text { Common } \\
\text { Chiffchaff }\end{array}$ & Phylloscopus collybita & C & & LC \\
\hline 149. & $\begin{array}{l}\text { Hume's Lesser } \\
\text { Whitethroat }\end{array}$ & Sylvia althaea & FC & & LC \\
\hline 150. & $\begin{array}{l}\text { Yellow Eyed } \\
\text { Babbler }\end{array}$ & Chrysomma sinense & C & & LC \\
\hline 151. & Common Babbler & Turdoides caudatus & FC & & LC \\
\hline 152. & $\begin{array}{l}\text { Large Grey } \\
\text { Babbler }\end{array}$ & Turdoides malcolmi & C & & LC \\
\hline 153. & Jungle Babbler & Turdoides straitus & C & & LC \\
\hline & Indian Bushlark & Mirafra erythroptera & FC & Alaudidae & LC \\
\hline 155. & Oriental Skylark & Alauda gulgula & C & & LC \\
\hline 156. & $\begin{array}{l}\text { Ashy-crowned } \\
\text { sparrow lark }\end{array}$ & Eremopterix grisea & FC & Alaudidae & LC \\
\hline 157. & Purple Sunbird & Nectarinia asiatica & C & Nectariniidae & LC \\
\hline 158. & $\begin{array}{c}\text { House Sparrow } \\
\text { Chestnut }\end{array}$ & Passer domesticus & C & & LC \\
\hline 159. & $\begin{array}{l}\text { Shouldered } \\
\text { Petronia }\end{array}$ & Petronia xanthocollis & FC & & LC \\
\hline 160. & White Wagtail & Motacilla personata & C & & LC \\
\hline 161. & Cristine Wagtail & Motacilla calcarata & C & & LC \\
\hline 162. & Yellow Wagtail & Motacilla thunbergi & C & & LC \\
\hline 163. & Grey Wagtail & Motacilla cinerea & FC & & LC \\
\hline 164. & Paddy field Pipit & Anthus rufulus & C & Passeridae & LC \\
\hline 165. & $\begin{array}{l}\text { Olive-backed } \\
\text { Pipit }\end{array}$ & Anthus hodgsoni & FC & & LC \\
\hline 166. & Baya Weaver & Ploceus philippinus & C & & LC \\
\hline 167. & $\begin{array}{l}\text { Black-breasted } \\
\text { weaver }\end{array}$ & Ploceus benghalensis & FC & & LC \\
\hline 168. & Red Avadavat & Amandava amandava & FC & & LC \\
\hline 169. & Indian Silverbill & Lonchura malabarica & C & & LC \\
\hline 170. & $\begin{array}{c}\text { Scaly Breasted } \\
\text { Munia }\end{array}$ & Lonchura punctulata & FC & & LC \\
\hline
\end{tabular}

C-Common; FC-Fairly Common; UC-Uncommon; NA-Not Assessed; LC-Least Concern; NT-Near Threatened; V-Vulnerable; E-Endangered 


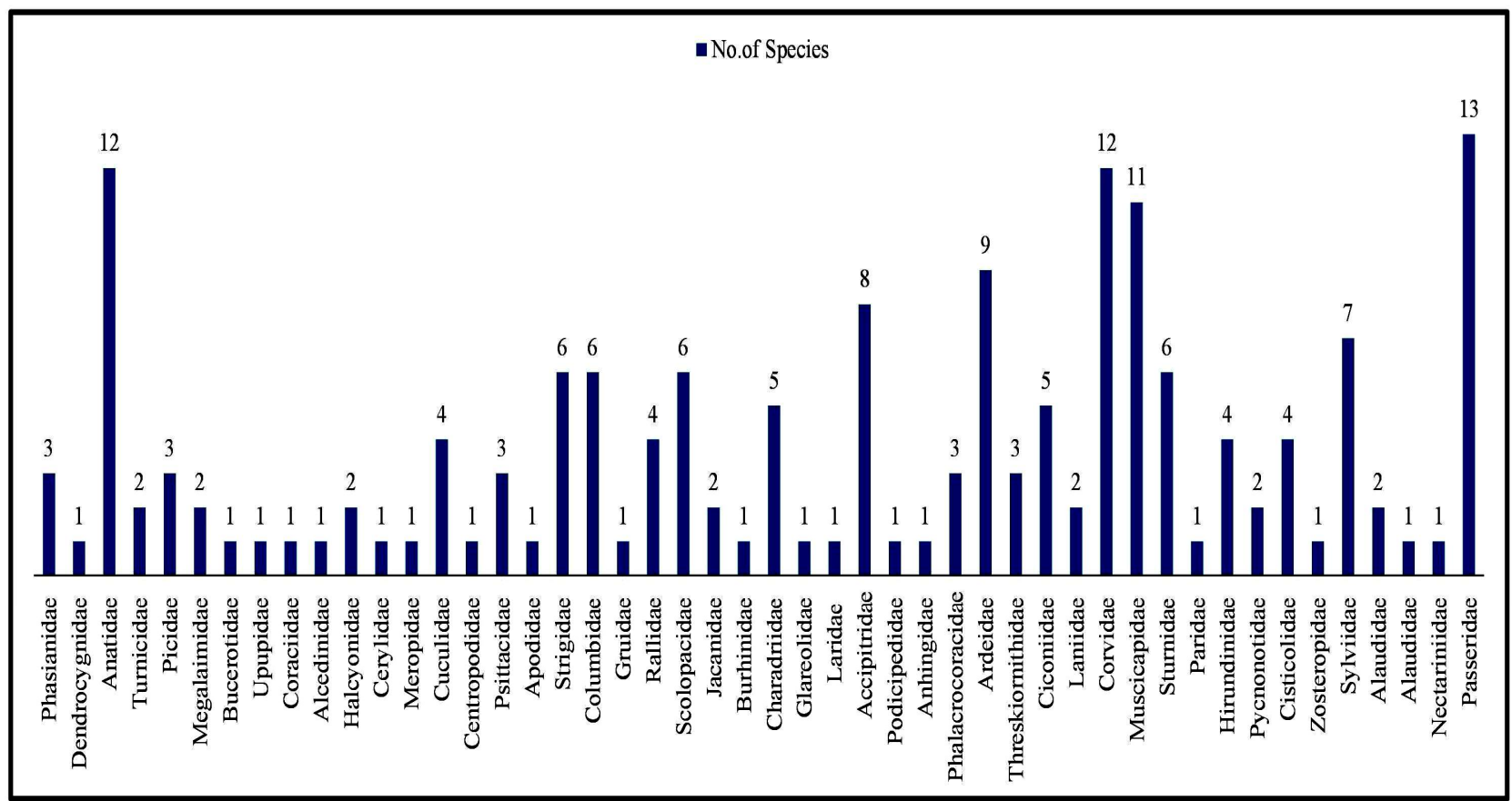

Fig. 2: Total of 170 bird species belonging to 48 families

Amongst the 170 species, 11 were Uncommon, 64 were fairly common and rest 95 species were common (Fig. $5 a-0)$, i.e only $6 \%$ of the birds were uncommon (Fig. 3). The least concerned category included 158 bird species (Fig. 4). According to the IUCN status, $1 \mathrm{sp}$. was endangered, 8 near Threatened, 1 Vulnerable. For 2 species, data was Not Available (Fig. 6a-f). The most common birds recorded were crows, House sparrows, Parakeets, Yellow-footed green pigeons, lapwings, munias, bulbuls, mynas and Baya weaver.

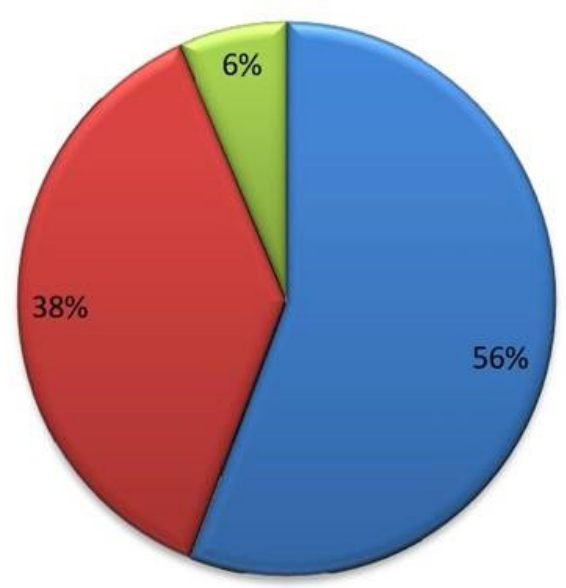

๑ Common

口 Fairly Common

$\square$ Uncommon

Abundance code

Fig. 3: Common, Fairly Common, Uncommon birds 


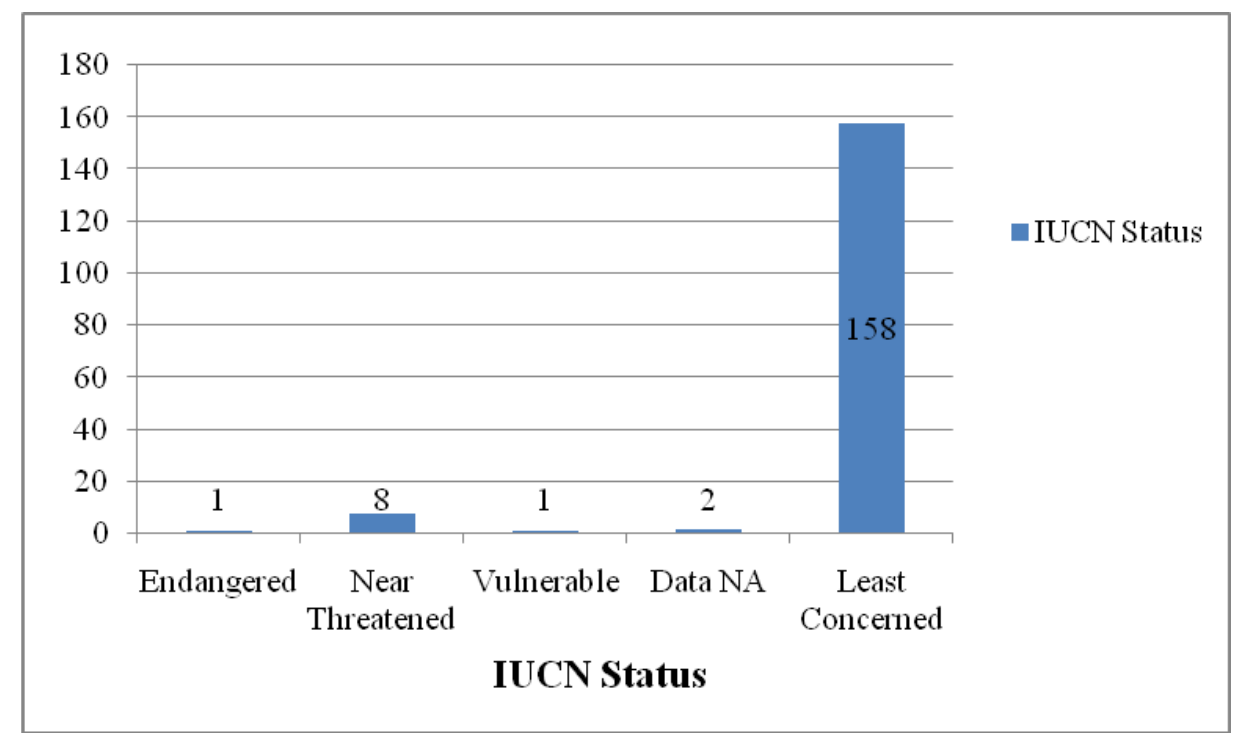

Fig. 4: IUCN Status of bird species

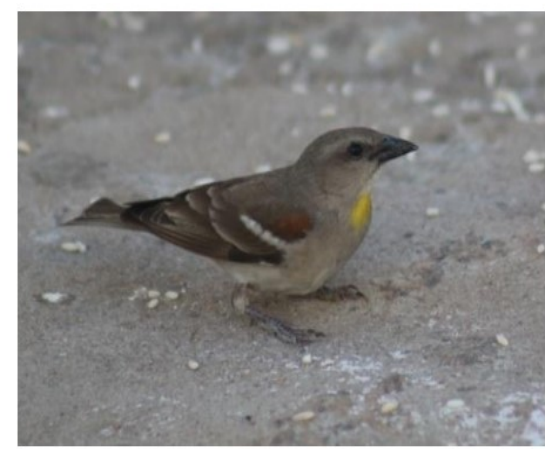

\section{a. Chestnut Shouldered Petronia}

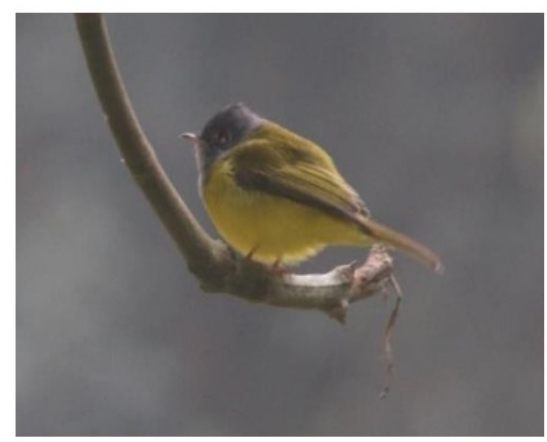

d. Grey-headed canary flycatcher

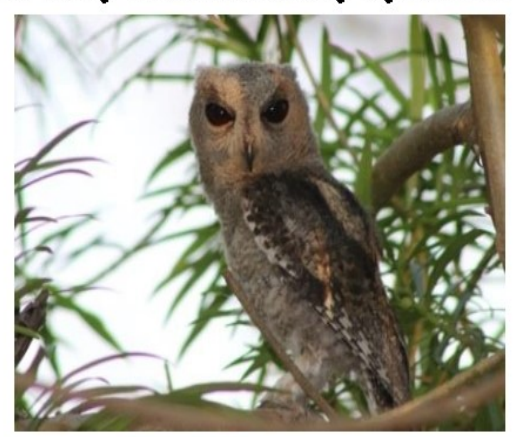

g. Collared Scoop's Owl

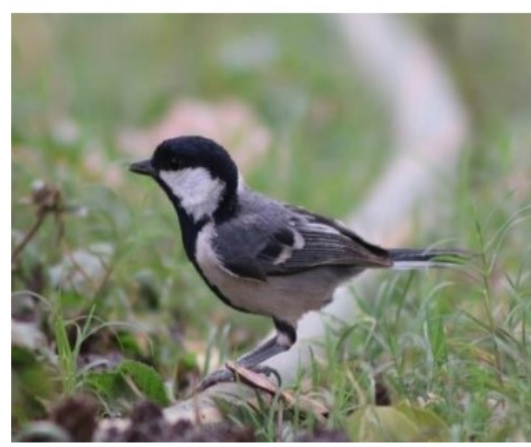

b.Great Tit

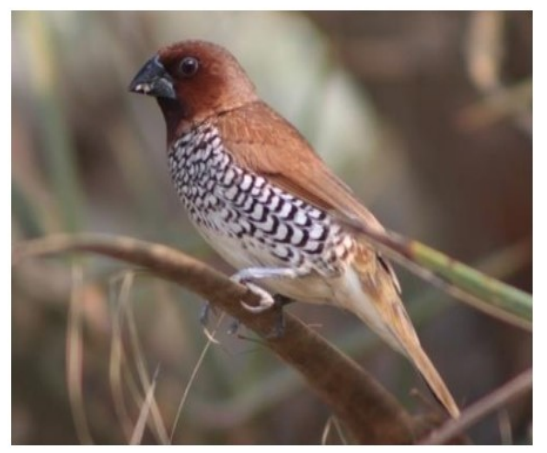

e.Scaly-breasted munia

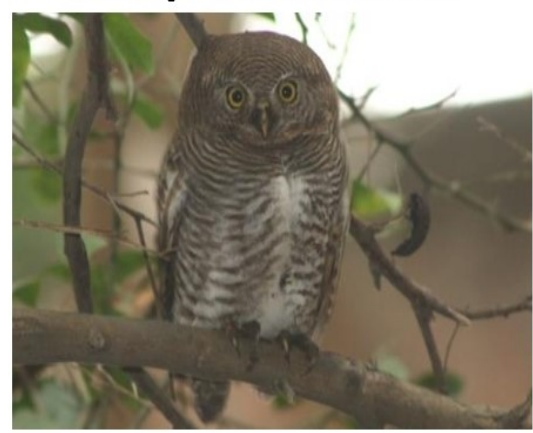

h. Jungle owlet

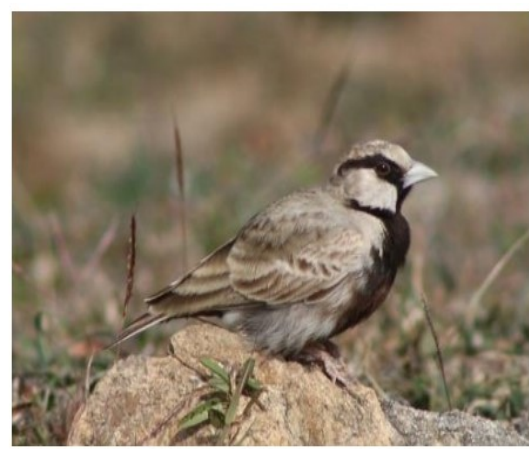

\section{c. Ashy-crowned sparrow lark}
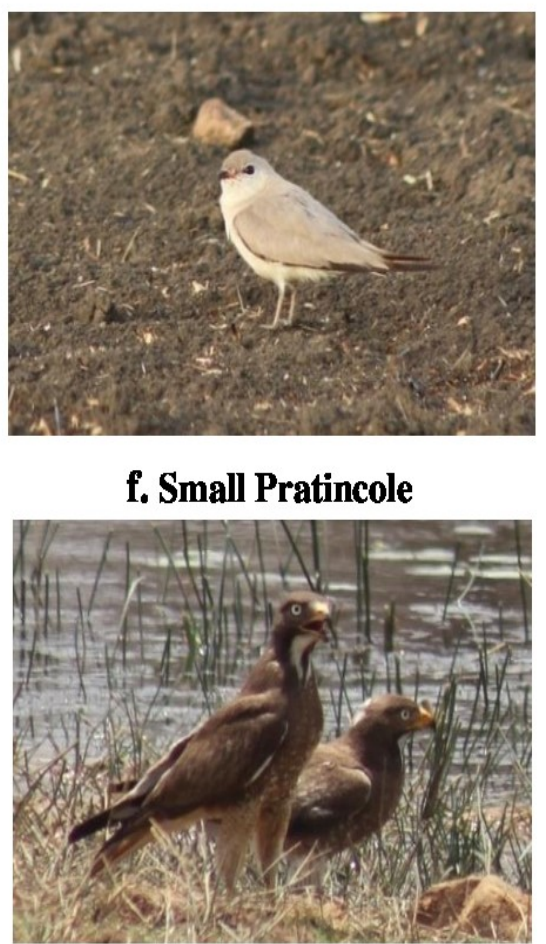

i. White eyed buzzard 

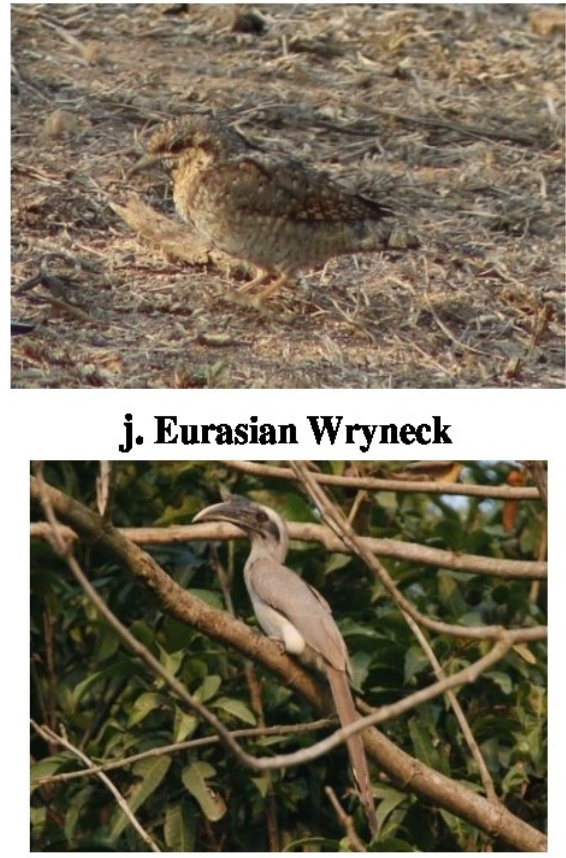

m. Grey Hornbill

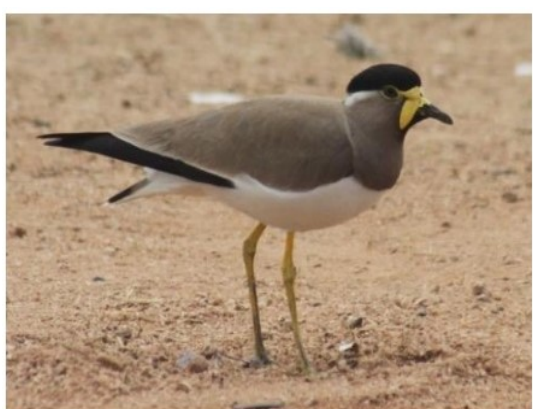

\section{k. Yellow wattled lapwing}

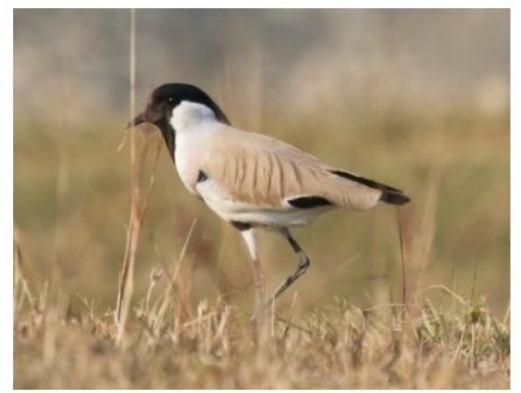

n. River Lapwing

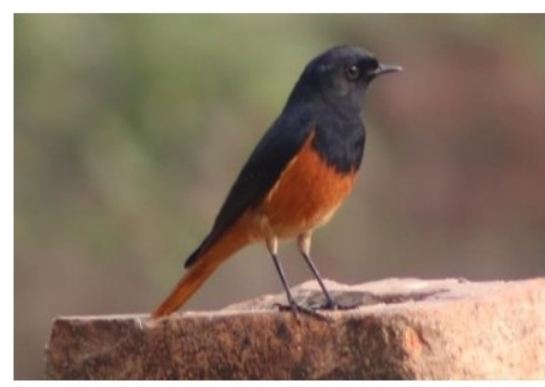

1. Red Blackstart

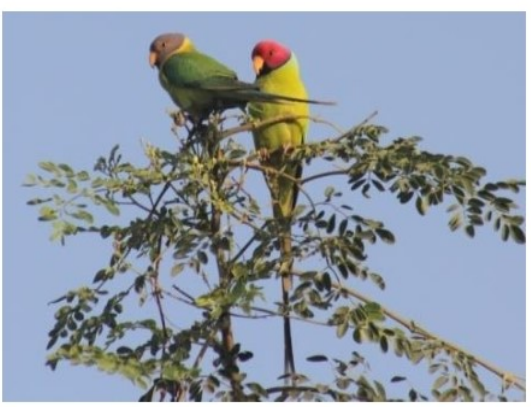

o. Plum-headed Parakeet

Fig. 5a-o: Some Uncommon and Fairly common birds in Ambedkar Nagar

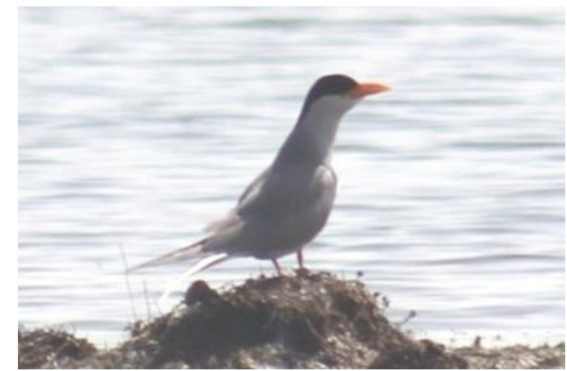

a. River Tern

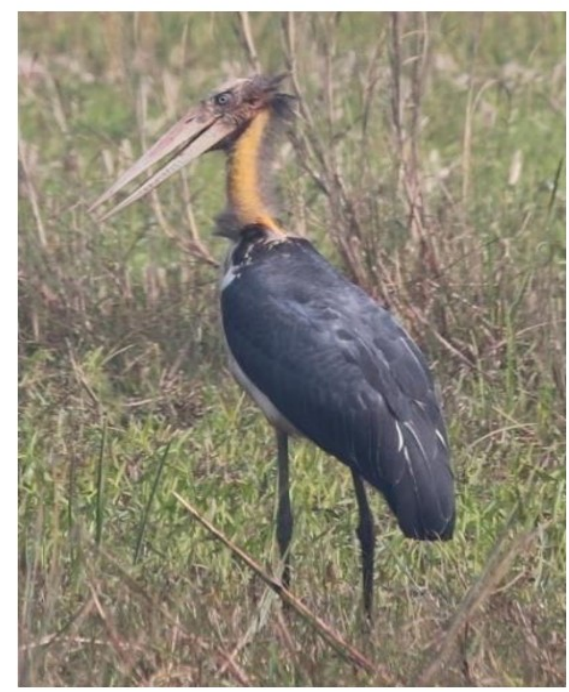

d. Lesser adjutant

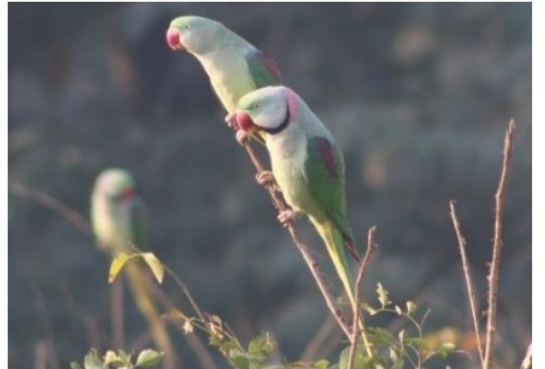

b. Alexandrine Parakeet

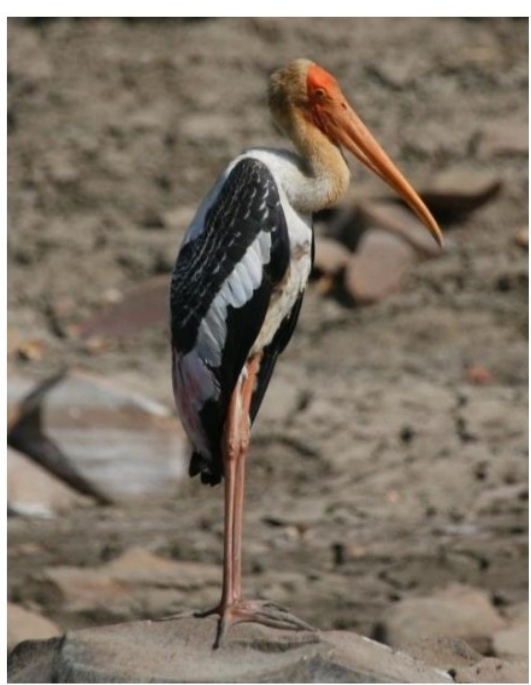

e. Painted Stork

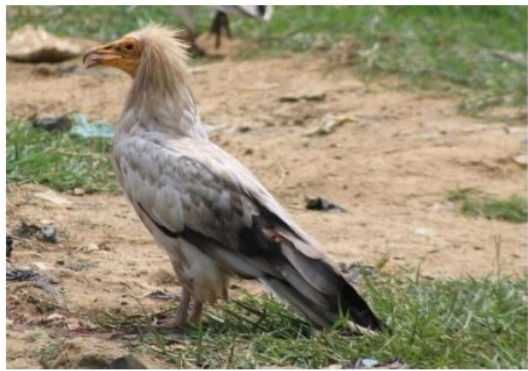

c. Egyptian Vulture

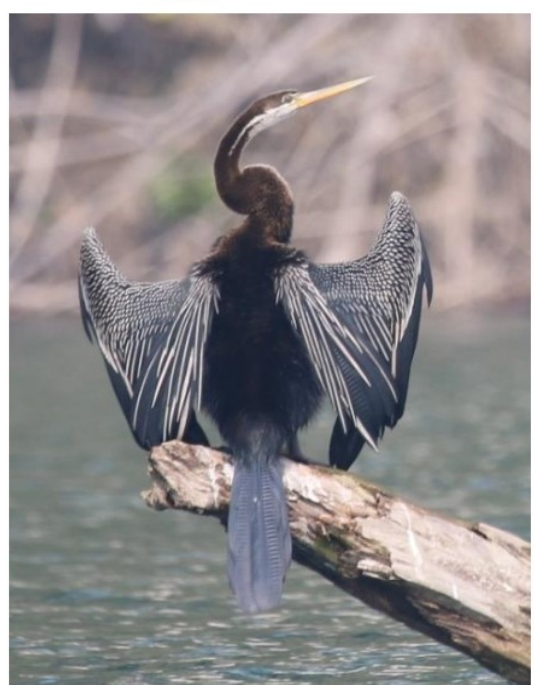

f. Darter

Fig. 6a-f: Some Endangered, Near Threatened and Vulnerable species 
The Indian National bird "Peacock" and the State bird of Uttar Pradesh "Sarus Crane" were easily spotted birds in almost all the tehsils of the district (Fig. 7a \& b). Though the Census for Sarus cranes undertaken in 2010 by Forest Department reported zero data for Ambedkar Nagar ${ }^{[10]}$, they were also seen nesting in the small local village ponds and also in rice fields. Similarly, no vultures had been reported in the district so far ${ }^{[11]}$, but the Endangered Egyptian Vultures were also sighted occasionally (Fig. 6c). The district had no dense forest coverage and hilly terrain, therefore, the birds of rocky and cliff habitats were not recorded during the study.

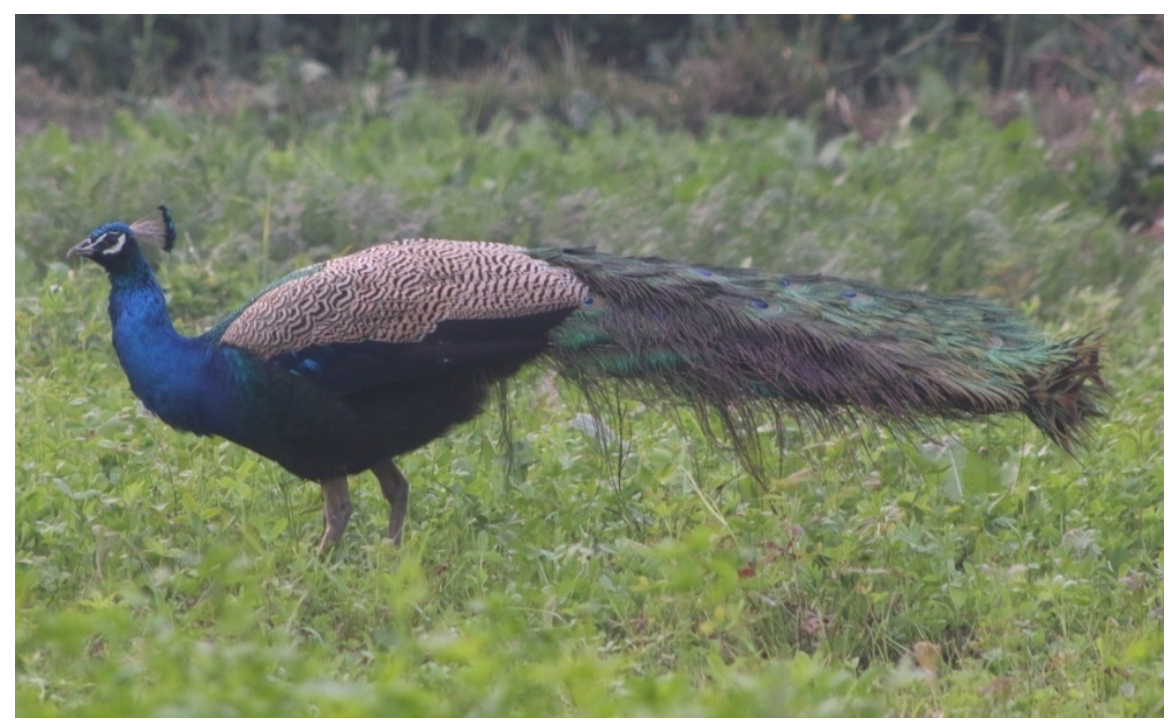

Fig. 7a: Indian National bird "Peacock"

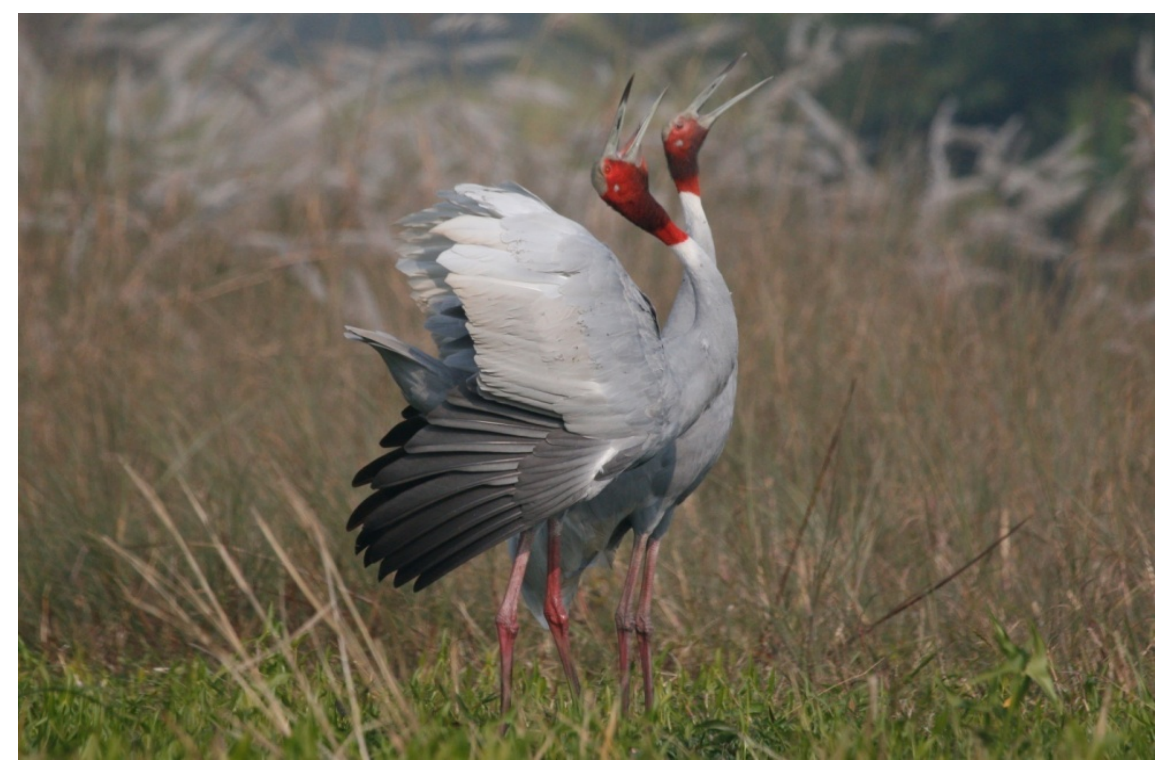

Fig. 7b: State bird of Uttar Pradesh "Sarus Crane"

\section{DISCUSSION}

Ambedkar Nagar, being an agricultural dominant area, the majority of birds reported was those that inhabit an agricultural landscape. There is growing interest in avian diversity in the agricultural area ${ }^{[12-18]}$. Such studies were useful in the management and conservation of useful bird species and control of pest birds. Sarus cranes were mostly observed in the crop fields and only a few pairs were seen in small unprotected local ponds. Due to the deterioration and destruction of natural wetland habitats, Sarus cranes were increasingly being forced into agricultural fields all over its distribution range in India ${ }^{[19]}$. Most of the districts have at least one or two locates that have important birding sites ${ }^{[20-22]}$; however, no particular site was discovered that could be identified as a birding spot in the district. The lakes such as Darvan, 
Hanswar, Devhat had the potential to support rich avifaunal diversity conversely they were under serious threats due to various anthropogenic activities. Various anthropogenic activities like uses of pesticides and insecticides in agriculture, deforestation, livestock grazing, hunting, fishing, development of industries and urbanization, sound pollution are some of the key threats to the avian diversity ${ }^{[23]}$. The majority of the aquatic birds were migratory; various types of ducks visit the lakes at the beginning of the winter. However, the numbers were low. The basic requirements of migratory birds at their wintering ground are adequate food supply and safety ${ }^{[24]}$, which was not fulfilled by the water bodies in Ambedkar Nagar. Intensification of agriculture and use of an excess of pesticides have severely affected the faunal diversity of the wetlands ${ }^{[25,26]}$. The wetlands being neglected by the local people and the concerned authorities may soon be lost forever.

\section{CONCLUSIONS}

The study reflects the potential of Ambedkar Nagar to support a rich diversity of ornithofauna. Further studies based on the ecology of threatened and endangered birds such as Egyptian Vultures, Sarus crane, Alexandrine Parakeet, Woolly-necked stork and many more species are needed. As for the conservation of habitat, the extremely low forest cover and anthropogenic activities around the wetlands are serious problems that need immediate concern and elucidation.

The district demands regular monitoring and reporting of incidences related to birds. There is a need to reduce anthropogenic mortality of birds or to educate the public to support for and to implement remedial measures. The organization of bird watching events on various occasions such as World Wetlands Day, International Day for Biological Diversity and World Wildlife Week will draw the intention and interest of local people and youth.

\section{ACKNOWLEDGMENTS}

The authors are thankful to the volunteers and local people for their kind support and cooperation during this much-needed study.

\section{CONTRIBUTION OF AUTHORS}

Research concept- Dr. Akhilesh Kumar

Research design- Dr. Akhilesh Kumar

Supervision- Dr. Sonika Kushwaha

Materials- Dr. Akhilesh Kumar
Data collection- Dr. Akhilesh Kumar

Data analysis and Interpretation- Dr. Sonika Kushwaha

Literature search- Dr. Akhilesh Kumar

Writing article- Dr. Akhilesh Kumar

Critical review- Dr. Sonika Kushwaha

Article editing- Dr. Sonika Kushwaha

Final approval- Dr. Akhilesh Kumar

\section{REFERENCES}

[1] Grimmett R, Inskipp C, Inskipp T. Birds of the Indian subcontinent. London: A \& C Black/Christopher Helm. Oxford University Press, 2011.

[2] Biodiversity: Living Treasures of Uttar Pradesh. Uttar Pradesh State Biodiversity Board: 2014; pp. 18.

[3] District statistical magazine, Ambedkar Nagar district, 1995-2012.

[4] District Planning Map. National Atlas and Thematic Mapping Organization, D.S.T., Kolkata, 2005.

[5] Ranjan V. District Ground Water Brochure. Ambedkar Nagar District, Uttar Pradesh, Central Ground Water Board Northern Region Lucknow January, 2014.

[6] Verner J. Assessment of counting techniques. Current Ornithology, 1985; 2: 247-302.

[7] Bibby CJ, Burgess ND, Hill DA, Mustoe SH. Bird census techniques. Academic Press, London, 2000.

[8] Ali S, Ripley SD. Compact handbook of the birds of India and Pakistan. Second edition. Oxford University Press, Bombay, 1983.

[9] Grimmett R, Inskipp C, Inskipp T. Birds of the Indian Subcontinent. $2^{\text {nd }}$ ed. London: Oxford University Press \& Christopher Helm., 2011; pp. 1-528.

[10]Jha KK, Singh P. Sarus Crane. State Bird of Uttar Pradesh. Bio. Div News, 2010; 1(5): 02-06.

[11] Jha KK. Distribution of vultures in Uttar Pradesh, India. Journal of Threatened Taxa, 2015; 7(1): 675063.

[12]Wang ZJ, Young SS. Differences in bird diversity between two swidden agricultural sites in mountainous terrain, Xishuangbanna, Yunnan, China. Bio. Conserv., 2003; 110: 231-43.

[13]Naidoo R. Species richness and community of songbirds in a tropical forest-agricultural landscape. Anim. Conserv., 2004; 7: 93-105.

[14]Waltert $M$, Bobo KS, Sainge $N H$, Fermon $H$, Muhlenberg M. From forest to farmland; habitat effect on Afrotropical forest bird diversity. Ecol. Appl., 2005; 15: 1351-66. 
[15]Perfecto I, Vandermeer J. Biodiversity conservation in tropical agro-ecosystems-A new conservation paradigm. Ann. NY Acad. Scip., 2008; 173-200.

[16]Clough Y, Barkmann J. Combining high biodiversity with high yield in tropical agroforests. Proc. Natl. Acad. Sci. USA, 2011; 108: 8311-16.

[17]Sekercioglu $\mathrm{CH}$. Bird functional diversity and ecosystem services in tropical forest, agroforests and agricultural area. J. Ornithol., 2010; 153-61.

[18]Abdar MR. Seasonal Diversity of Birds and Ecosystem Services in Agricultural Area of Western Ghats, Maharashtra State, India. IOSR J. Environ. Sci. Toxicol. Food Technol., 2014; 8(1): 100-05.

[19]Sundar KSG, Kaur J, Choudhury BC. Distribution, demography and conservation status of the Indian sarus Crane (Grus antigone) in India. J. Bombay Natl. Hist. Soc., 2000; 97(3): 319-39.

[20]Kushwaha S, Kumar D, et al. Avifaunal diversity of Govind Sagar dam in Lalitpur, Uttar Pradesh. Int. J. Res. Stud. Biosci., 2016; 5(4): 43-55.

[21]Kushwaha S, Kumar A, Namdev A. Assessment of Bird Community In Narayan Bagh, An Urban Park of Jhansi, Uttar Pradesh, India. Eco Chronicle, 2018; 13(3): 89-95.
[22] Lodhi RK, Rao RJ. Present status of aquatic bird diversity in Atal Sagar Dam Shivpuri Madhya Pradesh, India. Int. J. Adv. Res. Dev., 2017; 2(5): 10-13.

[23]Mistry J. Avifaunal diversity in and around Berhampore, Murshidabad district, West Bengal, India. Int. J. Fauna Biol. Stud., 2015; 2(4): 6-10.

[24]Lakshmi BB. Avifauna of Gosthani estuary near Visakhapatnam, Andhra Pradesh. J Nat. Conserv. 2006; 18(2): 291-364.

[25]Heard MS, Hawes C, Champion GT, Clark SJ, Firbank $L G$, et al. Weeds in fields with contrasting conventional and genetically modified herbicidetolerant crops. The effects on individual species. Philosophical Transactions of the Royal Society B. Biol. Sci., 2003; 358: 1833-46. doi: 10.1098/rstb.2003.1401.

[26]Joshi P, Krishna VK. Diversity of avifauna and effects of human activities on birds at Tawa Reservoir Area of Hoshangabad District (Madhya Pradesh) India. Adv. Res. Agri. Vet. Sci., 2014; 1(2): 78-82. 